TRANSACTIONS OF THE

AMERICAN MATHEMATICAL SOCIETY

Volume 362, Number 2, February 2010, Pages 727-751

S 0002-9947(09)04922-8

Article electronically published on July 29, 2009

\title{
THE DIMENSIONS OF A NON-CONFORMAL REPELLER AND AN AVERAGE CONFORMAL REPELLER
}

\author{
JUNGCHAO BAN, YONGLUO CAO, AND HUYI HU
}

\begin{abstract}
In this paper, using thermodynamic formalism for the sub-additive potential, upper bounds for the Hausdorff dimension and the box dimension of non-conformal repellers are obtained as the sub-additive Bowen equation. The map $f$ only needs to be $C^{1}$, without additional conditions. We also prove that all the upper bounds for the Hausdorff dimension obtained in earlier papers coincide. This unifies their results. Furthermore we define an average conformal repeller and prove that the dimension of an average conformal repeller equals the unique root of the sub-additive Bowen equation.
\end{abstract}

\section{INTRODUCTION}

In the dimension theory of dynamical systems, it is a very interesting topic to study the Hausdorff dimension of invariant sets of hyperbolic dynamics. Bowen [3] was the first to express the Hausdorff dimension of an invariant set as a solution of an equation involving topological pressure. Ruelle 13 refined Bowen's method and obtained the following result. Assume that $f$ is a $C^{1+\gamma}$ conformal expanding map, $\Lambda$ is an isolated compact invariant set and $\left.f\right|_{\Lambda}$ is topologically mixing. Then the Hausdorff dimension of $\Lambda, \operatorname{dim}_{H} \Lambda$, is given by the unique solution $\alpha$ of the equation

$$
P\left(\left.f\right|_{\Lambda},-\alpha \log \left\|D_{x} f\right\|\right)=0,
$$

where $P\left(\left.f\right|_{\Lambda}, \cdot\right)$ is the topological pressure functional. The smoothness $C^{1+\gamma}$ was recently relaxed to $C^{1}$ [9].

An estimate from above for the Hausdorff dimension of compact invariant sets for differentiable maps has been given by A. Douady and J. Oesterlé [5], and by Ledrappier [11. For non-conformal dynamical systems there exist only partial results. For example, the Hausdorff dimension of hyperbolic invariant sets was only computed in some special cases. $\mathrm{Hu}$ [10, gave an estimate of the dimension of the non-conformal repeller for a $C^{2}$ map. Falconer [6, 7] computed the Hausdorff dimension of a class of non-conformal repellers. Related ideas were applied by Simon and Solomyak 16 to compute the Hausdorff dimension of a class of non-conformal horseshoes in $R^{3}$.

Received by the editors November 6, 2007.

2000 Mathematics Subject Classification. Primary 37D35; Secondary 37C45.

Key words and phrases. Hausdorff dimension, non-conformal repellers, topological pressure.

Yongluo Cao is the corresponding author. 
For $C^{1}$ non-conformal repellers, in [18, Zhang uses singular values of the derivative $D_{x} f^{n}$ for all $n \in Z^{+}$to define a new equation which involves the limit of a sequence of topological pressure. Then he shows that the unique solution of the equation is an upper bound for the Hausdorff dimension of the repeller. In [1, the same problem is considered. Barreira bases his estimates on the non-additive thermodynamic formalism which was introduced in 2 and the singular value of the derivative $D_{x} f^{n}$ for all $n \in Z^{+}$, and gives an upper bound for the box dimension of the repeller under the additional assumptions for which the map is $C^{1+\gamma}$ and $\gamma$-bunched. This automatically implies that for Hausdorff dimension. In 8 , Falconer defines topological pressure of sub-additive potential under the condition $\left\|\left(D_{x} f\right)^{-1}\right\|^{2}\left\|D_{x} f\right\|<1$, which means that $f$ is 1 -bunched. They also obtain an upper bound for the Hausdorff dimension of the repeller. The questions are whether the three bounds as above are the same and whether the upper bounds of the box dimension hold true for a $C^{1}$ non-conformal repeller.

In this paper, the first, using thermodynamic formalism for sub-additive potential defined in [4, we can obtain upper bounds for the Hausdorff dimension and the box dimension of non-conformal repellers. The map $f$ only needs to be $C^{1}$, without additional conditions. In fact, we prove that the upper bound of the Hausdorff dimension of non-conformal repellers in [18 is the unique root of the generalized Bowen equation which relates to sub-additive thermodynamic formalism. Furthermore, we proved all the upper bounds in 1, 18, 8, and ours are the same and we can prove that topological pressure in [4] is the same as in [1, 8] in which they need that $f$ is $C^{1+\gamma}$ and $\gamma$-bunched. Our result also gives an affirmative answer to a problem posed by K. Simon in [15] concerning an upper bound without assuming the 1-bunched property.

Then we introduce the notion of an average conformal repeller. Using thermodynamic formalism for sub-additive potential, we prove that the Hausdorff dimension and the box dimension of average conformal repellers equal the unique root of the Bowen equation for sub-additive topological pressure. The map $f$ only needs to be $C^{1}$, without additional conditions. Meanwhile, we introduce super-additive potential topological pressure and prove that for special potentials, sub-additive and super-additive topological pressures are the same. In [2, Barreira introduces the concept of a quasi-conformal repeller by using Markov construction and proves that its dimension is the unique root of the equation obtained by non-additive topological pressure. In 12 is introduced the concept of a weakly conformal repeller and its dimension using the Bowen equation is obtained. It is obvious that for $C^{1}$ maps, quasi-conformal and weakly conformal repellers are average conformal repellers, but the reverse is not true. Therefore our result is a generalization of the results in 2, 12 .

Next we recall some basic definitions and notation.

Let $f: X \rightarrow X$ be a continuous map. A set $E \subset X$ is called an $(n, \epsilon)$-separated set with respect to $f$ if whenever $x, y \in E$, then $d_{n}(x, y)=\max _{0 \leq i \leq n-1} d\left(f^{i} x, f^{i} y\right)$ $>\epsilon$. For $x \in X$ and $r>0$, define

$$
B_{n}(x, r)=\left\{y \in X: f^{i} y \in B\left(f^{i} x, r\right) \text {, for all } i=0, \ldots, n-1\right\} .
$$

If $\phi$ is a real continuous function on $X$ and $n \in Z^{+}$, let

$$
S_{n} \phi(x)=\sum_{i=0}^{n-1} \phi\left(f^{i}(x)\right) .
$$


We define

$$
P_{n}(\phi, \epsilon)=\sup \left\{\sum_{x \in E} \exp S_{n} \phi(x): E \text { is an }(n, \epsilon) \text {-separated subset of } X\right\} .
$$

Then the topological pressure of $\phi$ is given by

$$
P(f, \phi)=\lim _{\epsilon \rightarrow 0} \limsup _{n \rightarrow \infty} \frac{1}{n} \log P_{n}(\phi, \epsilon) .
$$

Next we give some properties of $P(f, \cdot): C(M, R) \rightarrow R \cup\{\infty\}$.

Proposition 1.1. Let $f: M \rightarrow M$ be a continuous transformation of a compact metrisable space $M$. If $\varphi_{1}, \varphi_{2} \in C(X, R)$, then the following are true:

(1) $P(f, 0)=h_{t o p}(f)$.

(2) $\left|P\left(f, \varphi_{1}\right)-P\left(f, \varphi_{2}\right)\right| \leq\left\|\varphi_{1}-\varphi_{2}\right\|$.

(3) $\varphi_{1} \leq \varphi_{2}$ implies that $P\left(f, \varphi_{1}\right) \leq P\left(f, \varphi_{2}\right)$.

Proof. See Walters' book 17.

Corollary 1. Let $f: M \rightarrow M$ be a continuous transformation of a compact metrisable space $M$. If $\varphi \in C(M, R)$ and $\varphi<0$, then the function $P(\alpha)=P(f, \alpha \varphi)$ is continuous and strictly decreasing in $\alpha$.

Proof. Let $M=\max _{x \in M} \varphi(x)$ and $m=\min _{x \in M} \varphi(x)$. Then $\varphi \in C(M, R)$ and $\varphi<0$ imply that $m \leq M<0$. If $\alpha_{1}<\alpha_{2}$, then for all $n \in \mathbb{N}$, we have

$$
\left(\alpha_{2}-\alpha_{1}\right) n m \leq S_{n}\left(\alpha_{2} \varphi\right)(x)-S_{n}\left(\alpha_{1} \varphi\right)(x)=\left(\alpha_{2}-\alpha_{1}\right) S_{n} \varphi(x) \leq\left(\alpha_{2}-\alpha_{1}\right) n M .
$$

Thus for $\forall \epsilon>0$,

$$
e^{\left(\alpha_{2}-\alpha_{1}\right) n m} \times P_{n}\left(\alpha_{1} \varphi, \epsilon\right) \leq P_{n}\left(\alpha_{2} \varphi, \epsilon\right) \leq P_{n}\left(\alpha_{1} \varphi, \epsilon\right) \times e^{\left(\alpha_{2}-\alpha_{1}\right) n M} .
$$

This implies that

$$
\left(\alpha_{2}-\alpha_{1}\right) m+P\left(f, \alpha_{1} \varphi\right) \leq P\left(f, \alpha_{2} \varphi\right) \leq P\left(f, \alpha_{1} \varphi\right)+\left(\alpha_{2}-\alpha_{1}\right) M .
$$

Therefore $P(f, \alpha \varphi)$ is continuous and strictly monotone decreasing on $\alpha$.

Another equivalent definition of topological pressure involves open covers.

Definition 1.1. If $\varphi \in C(M, R), n \geq 1$ and $\mathcal{U}$ is an open cover of $M$ put

$$
p_{n}(f, \phi, \mathcal{U})=\inf \left\{\sum_{\beta} \sup _{x \in B} e^{S_{n} \phi(x)} \mid \beta \text { is a finite subcover of } \bigvee_{i=0}^{n-1} f^{-i} \mathcal{U}\right\} .
$$

It is proved [17] that the limit

$$
\lim _{n \rightarrow \infty} \frac{1}{n} \log p_{n}(f, \phi, \mathcal{U})
$$

exists and is equal to $\inf _{n>0}\left\{\frac{1}{n} \log p_{n}(f, \varphi, \mathcal{U})\right\}$.

We have the following lemma whose proof can be found in [17].

Lemma 1.1. If $\phi \in C(M, R), n \geq 1$ and $\mathcal{U}$ is an open cover of $M$, then

$$
\lim _{\operatorname{diam}(\mathcal{U}) \rightarrow 0} \lim _{n \rightarrow \infty} \frac{1}{n} \log p_{n}(f, \phi, \mathcal{U})=P(f, \phi) .
$$


A linear map $L: \mathbb{R}^{n} \rightarrow \mathbb{R}^{n}$ is said to be expanding if $\|L v\|>\|v\|$ for all $v \in \mathbb{R}^{n}$ and $v \neq 0$. Given an expanding linear map $L: \mathbb{R}^{m} \rightarrow \mathbb{R}^{m}$, let $\lambda_{1} \geq \lambda_{2} \geq \cdots \geq$ $\lambda_{m} \geq 0$ be the logarithms of the singular values of $L$, which are eigenvalues of $\left(L^{*} L\right)^{\frac{1}{2}}$, counted with their multiplicities, where $\lambda_{m}>0$ because of the expansion. Following [5] we introduce the function

$$
g^{\alpha}(L)=\sum_{i=m-[\alpha]+1}^{m} \lambda_{i}+(\alpha-[\alpha]) \lambda_{m-[\alpha]},
$$

for any $\alpha \in[0, m]$, where $[\alpha]$ is the largest integer $\leq \alpha . g^{\alpha}(L)$ is continuous and strictly increasing in $\alpha \cdot g^{0}(L)=0$ and $g^{m}(L)=\sum_{i=1}^{m} \lambda_{i}=\log |\operatorname{Jac}(L)|$, where $\operatorname{Jac}(L)$ is the Jacobian of $L$. The map $g^{\alpha}$ has the following super-additive property. If $L: \mathbb{R}^{m} \rightarrow \mathbb{R}^{m}$ and $L^{\prime}: \mathbb{R}^{m} \rightarrow \mathbb{R}^{m}$ are two expanding maps, then

$$
g^{\alpha}\left(L^{\prime} L\right) \geq g^{\alpha}\left(L^{\prime}\right)+g^{\alpha}(L) .
$$

The paper is organized as follows. In Section 2, we develop the sub-additive thermodynamic formalism and prove that the upper bound of the Hausdorff dimension of non-conformal repellers in [18] is exactly the unique root of the equation of sub-additive topological pressure. In Section 3, we consider the relation between sub-additive thermodynamic formalism defined in [4] and [2, 8], and we obtain for a $C^{1}$ non-conformal repeller $\Lambda$, that the upper box dimension is bounded by a value which is the unique solution of the equation of sub-additive topological pressure. This is a generalization of the result in [2]. In Section 4, we introduce the definition of an average conformal repeller and give related results and the main theorem. In Section 5, we develop super-additive thermodynamic formalism and the variational principle for super-additive potential. In Section 6 , we give the proof of the main result.

\section{A SUB-ADDITIVE THERMODYNAMIC FORMALISM}

Let $f: X \rightarrow X$ be a continuous map. A set $E \subset X$ is called an $(n, \epsilon)$-separated set with respect to $f$ if whenever $x, y \in E$, then $d_{n}(x, y)=\max _{0 \leq i \leq n-1} d\left(f^{i} x, f^{i} y\right)$ $>\epsilon$. A sub-additive valuation on $X$ is a sequence of continuous functions $\phi_{n}: M \rightarrow$ $R$ such that

$$
\phi_{m+n}(x) \leq \phi_{n}(x)+\phi_{m}\left(f^{n}(x)\right)
$$

we denote it by $\mathcal{F}=\left\{\phi_{n}\right\}$.

In the following we will define the topological pressure of $\mathcal{F}=\left\{\phi_{n}\right\}$ with respect to $f$. We define

$$
P_{n}(\mathcal{F}, \epsilon)=\sup \left\{\sum_{x \in E} \exp \phi_{n}(x): E \quad \text { is an }(n, \epsilon) \text {-separated subset of } X\right\} .
$$

Then the topological pressure of $\mathcal{F}$ is given by

$$
P(f, \mathcal{F})=\lim _{\epsilon \rightarrow 0} \limsup _{n \rightarrow \infty} \frac{1}{n} \log P_{n}(\mathcal{F}, \epsilon) .
$$

Let $\mathcal{M}(X)$ be the space of all Borel probability measures endowed with the weak* topology. Let $\mathcal{M}(X, f)$ denote the subspace of $\mathcal{M}(X)$ consisting of all $f$-invariant 
measures. For $\mu \in \mathcal{M}(X, f)$, let $h_{\mu}(f)$ denote the entropy of $f$ with respect to $\mu$, and let $\mathcal{F}_{*}(\mu)$ denote the following limit:

$$
\mathcal{F}_{*}(\mu)=\lim _{n \rightarrow \infty} \frac{1}{n} \int \phi_{n} d \mu .
$$

The existence of the above limit follows from a sub-additive argument. We call $\mathcal{F}_{*}(\mu)$ the Lyapunov exponent of $\mathcal{F}$ with respect to $\mu$ since it describes the exponential growth speed of $\phi_{n}$ with respect to $\mu$.

In [4, the authors proved the following variational principle.

Theorem 2.1 (4]). Under the above general setting, we have

$$
P(f, \mathcal{F})=\sup \left\{h_{\mu}(T)+\mathcal{F}_{*}(\mu): \mu \in \mathcal{M}(X, f)\right\} .
$$

In 2, Barreira used a different method to introduce topological pressure for sub-additive potential functions and proved the variational principle if the potential functions satisfy further conditions.

Let $M$ be a $C^{\infty}$ Riemann manifold, $\operatorname{dim} M=m$. Let $U$ be an open subset of $M$ and let $f: U \rightarrow M$ be a $C^{1}$ map. Suppose $\Lambda \subset U$ is a compact invariant set on which $f$ is expanding, that is, $f \Lambda=\Lambda$ and there is $k>1$ such that for all $x \in \Lambda$ and $v \in T_{x} M$,

$$
\left\|D_{x} f v\right\| \geq k\|v\|
$$

where $\|$.$\| is the norm induced by an adapted Riemannian metric. Let \mathcal{M}\left(\left.f\right|_{\Lambda}\right)$ denote all the $f$ invariant measures supported on $\Lambda$.

If $x \in \Lambda$, then $D_{x} f: T_{x} M \rightarrow T_{f x} M$ is a linear map. Denote the logarithms of the singular values of $D_{x} f$ by

$$
\lambda_{1}(x, f) \geq \lambda_{2}(x, f) \geq \cdots \geq \lambda_{m}(x, f) \geq \log k
$$

and for $\alpha \in[0, m]$, write

$$
g^{\alpha}(x, f)=g^{\alpha}\left(D_{x} f\right)=\sum_{i=m-[\alpha]+1}^{m} \lambda_{i}(x, f)+(\alpha-[\alpha]) \lambda_{m-[\alpha]}(x, f) .
$$

Since $f$ is $C^{1}$, the functions $x \mapsto \lambda_{i}(x, f)$ and $x \mapsto g^{\alpha}(x, f)$ are all continuous.

In fact, $f \Lambda=\Lambda$ implies $f^{n} \Lambda=\Lambda$. $f^{n}$ is also expanding on $\Lambda$. Let the logarithms of the singular value of $D_{x} f^{n}$ be

$$
\lambda_{1}\left(x, f^{n}\right) \geq \lambda_{2}\left(x, f^{n}\right) \geq \cdots \geq \lambda_{m}\left(x, f^{n}\right) \geq n \log k
$$

and set

$$
g^{\alpha}\left(x, f^{n}\right)=g^{\alpha}\left(D_{x} f^{n}\right)=\sum_{i=m-[\alpha]+1}^{m} \lambda_{i}\left(x, f^{n}\right)+(\alpha-[\alpha]) \lambda_{m-[\alpha]}\left(x, f^{n}\right) .
$$

The functions $g^{\alpha}\left(\cdot, f^{n}\right)$ satisfy

$$
g^{\alpha}\left(x, f^{n+l}\right) \geq g^{\alpha}\left(x, f^{n}\right)+g^{\alpha}\left(f^{n}(x), f^{l}\right) .
$$

Define a sequence of functions $P_{n}:[0, m] \rightarrow R$ as follows:

$$
P_{n}(\alpha)=P\left(\left.f\right|_{\Lambda},-\frac{1}{n} g^{\alpha}\left(\cdot, f^{n}\right)\right) .
$$

In [18], the author proved the following result. 
Lemma 2.1 ([18]). For every $\alpha \in[0, m]$, the following limit exists:

$$
\lim _{n \rightarrow \infty} P_{n}(\alpha)=\inf _{n \in Z^{+}} P_{n}(\alpha) .
$$

Set $P^{*}(\alpha)=\lim _{n \rightarrow \infty} P_{n}(\alpha)$. Then $P^{*}$ is continuous and strictly decreasing on $[0, m]$.

Theorem $2.2([18])$. Let

$$
\mathcal{D}(f, \Lambda)=\max \left\{\alpha \in[0, m]: P^{*}(\alpha) \geq 0\right\} .
$$

Then

$$
\operatorname{dim}_{H} \Lambda \leq \mathcal{D}(f, \Lambda) .
$$

Remark 1. By the variational principle and Ruelle's inequality, we have $P^{*}(m) \leq 0$. Since $P^{*}(0)=h\left(\left.f\right|_{\Lambda}\right)>0$, by Lemma 2.1, it follows that the equation $P^{*}(\alpha)=0$ has a unique solution on $[0, m]$. By the definition, we have that $\mathcal{D}(f, \Lambda)$ is the unique solution of the equation $P^{*}(\alpha)=0$.

In this paper, we first prove the following proposition.

Proposition 2.1. Suppose $\left\{\phi_{n}(x)\right\}$ is a sub-additive continuous function sequence on $M$. Let $\mathcal{F}=\left\{\phi_{n}\right\}$. Then we have $P(f, \mathcal{F})=\lim _{n \rightarrow \infty} P\left(f, \frac{\phi_{n}}{n}\right)$.

Proof. The existence of the limit $\lim _{n \rightarrow \infty} P\left(f, \frac{\phi_{n}}{n}\right)$ can be found in [18].

First we prove that

$$
P(f, \mathcal{F}) \leq \lim _{n \rightarrow \infty} P\left(f, \frac{\phi_{n}}{n}\right) .
$$

For a fixed $m$, let $n=m s+l, 0 \leq l<m$. From the subadditivity of $\left\{\phi_{n}\right\}$, we have

$$
\phi_{n}(x) \leq \frac{1}{m} \sum_{j=0}^{m-1} \sum_{i=0}^{s-2} \phi_{m}\left(f^{i m+j}(x)\right)+\frac{1}{m} \sum_{j=0}^{m-1}\left[\phi_{j}(x)+\phi_{m-j+l}\left(f^{(s-1) m+j}(x)\right)\right] .
$$

Let $C_{1}=\max _{i=1, \cdots, 2 m-1} \max _{x \in X} \phi_{i}(x)$. Then we have

$$
\begin{aligned}
\phi_{n}(x) & \leq \sum_{j=0}^{(s m+l)-1} \frac{1}{m} \phi_{m}\left(f^{j}(x)\right)-\frac{1}{m} \sum_{j=(s-1) m}^{s m-1} \phi_{m}\left(f^{j}(x)\right)+2 C_{1} \\
& \leq \sum_{j=0}^{n-1} \frac{1}{m} \phi_{m}\left(f^{j}(x)\right)+4 C_{1} .
\end{aligned}
$$

Hence we have

$$
\exp \left(\phi_{n}(x)\right) \leq \exp \left(\sum_{j=0}^{n-1} \frac{1}{m} \phi_{m}\left(f^{j}(x)\right)+4 C_{1}\right)
$$

Thus

$$
\begin{aligned}
P_{n}(\mathcal{F}, \epsilon) & =\sup \left\{\sum_{x \in E} \exp \phi_{n}(x): E \text { is an }(n, \epsilon) \text {-separated subset of } X\right\} \\
& \leq P_{n}\left(\frac{1}{m} \phi_{m}, \epsilon\right) \times \exp \left(4 C_{1}\right) .
\end{aligned}
$$

This implies

$$
P(f, \mathcal{F}) \leq P\left(f, \frac{1}{m} \phi_{m}\right) .
$$


From the arbitrariness of $m \in Z^{+}$, we have

$$
P(f, \mathcal{F}) \leq P\left(f, \frac{1}{m} \phi_{m}\right), \text { for all } m \in Z^{+} .
$$

Therefore

$$
P(f, \mathcal{F}) \leq \lim _{n \rightarrow \infty} P\left(f, \frac{\phi_{n}}{n}\right)
$$

Next, we prove that

$$
P(f, \mathcal{F}) \geq \lim _{n \rightarrow \infty} P\left(f, \frac{\phi_{n}}{n}\right) .
$$

Since $f: \Lambda \rightarrow \Lambda$ is an expanding map, $h_{\mu}(f)$ is an upper-semi-continuous function from $\mathcal{M}\left(\left.f\right|_{\Lambda}\right)$ to $R$. From the variational principle of topological pressure [17, we have that for every $k \in Z^{+}$there exists $\mu_{2^{k}} \in \mathcal{M}\left(\left.f\right|_{\Lambda}\right)$ such that

$$
P\left(\left.f\right|_{\Lambda}, \frac{1}{2^{k}} \phi_{2^{k}}\right)=h_{\mu_{2^{k}}}(f)+\int_{\Lambda} \frac{1}{2^{k}} \phi_{2^{k}} d \mu_{2^{k}} .
$$

Since $\mathcal{M}\left(\left.f\right|_{\Lambda}\right)$ is compact, this implies that $\mu_{2^{k}}$ has a subsequence which converges to $\mu \in \mathcal{M}\left(\left.f\right|_{\Lambda}\right)$. Without loss of generality, suppose that $\mu_{2^{k}}$ converges to $\mu$. Using the sub-additivity and invariance of $\mu_{2^{k}}$, then we have for every $k \in \mathbb{N}$,

$$
h_{\mu_{2^{k}}}(f)+\int_{\Lambda} \frac{\phi_{2^{k}}(x)}{2^{k}} d \mu_{2^{k}} \leq h_{\mu_{2^{k}}}(f)+\int_{\Lambda} \phi_{1}(x) d \mu_{2^{k}} .
$$

Furthermore for fixed $s \in \mathbb{N}$, if $k>s$, from the sub-additivity and invariance of $\mu_{2^{k}}$, we have

$$
h_{\mu_{2^{k}}}(f)+\int_{\Lambda} \frac{\phi_{2^{k}}(x)}{2^{k}} d \mu_{2^{k}} \leq h_{\mu_{2^{k}}}(f)+\int_{\Lambda} \frac{\phi_{2^{s}}(x)}{2^{s}} d \mu_{2^{k}} .
$$

Since $h_{\mu}(f)$ is an upper-semi-continuous function, we have

$$
\begin{aligned}
\lim _{n \rightarrow \infty} P\left(f, \frac{\phi_{n}}{n}\right) & =\lim _{k \rightarrow \infty} P\left(f, \frac{\phi_{2^{k}}}{2^{k}}\right) \\
& =\lim _{k \rightarrow \infty}\left(h_{\mu_{2^{k}}}(f)+\int_{\Lambda} \frac{\phi_{2^{k}}(x)}{2^{k}} d \mu_{2^{k}}\right) \\
& \leq \lim _{k \rightarrow \infty}\left(h_{\mu_{2^{k}}}(f)+\int_{\Lambda} \frac{\phi_{2^{s}}(x)}{2^{s}} d \mu_{2^{k}}\right) \\
& \leq h_{\mu}(f)+\int_{\Lambda} \frac{\phi_{2^{s}}(x)}{2^{s}} d \mu .
\end{aligned}
$$

Since sequence $\left\{\int_{\Lambda} \phi_{n}(x) d \mu\right\}$ is a sub-additive sequence, we have

$$
\lim _{n \rightarrow \infty} \int_{\Lambda} \frac{\phi_{n}(x)}{n} d \mu=\inf _{n \geq 1}\left\{\int_{\Lambda} \frac{\phi_{n}(x)}{n} d \mu\right\} .
$$

The arbitrariness of $s \in \mathbb{N}$ implies that

$$
\lim _{n \rightarrow \infty} P\left(f, \frac{\phi_{n}}{n}\right) \leq h_{\mu}(f)+\lim _{s \rightarrow \infty} \int_{\Lambda} \frac{\phi_{2^{s}}}{2^{s}}(x) d \mu .
$$

Hence by the variational principle of the sub-additive topological pressure in [4], we have

$$
\lim _{n \rightarrow \infty} P\left(f, \frac{\phi_{n}}{n}\right) \leq h_{\mu}(f)+\lim _{s \rightarrow \infty} \int_{\Lambda} \frac{\phi_{2^{s}}}{2^{s}}(x) d \mu \leq P(f, \mathcal{F}) .
$$

This completes the proof of Proposition 2.1 
Theorem 2.3. Let $\mathcal{F}(\alpha)=\left\{-g^{\alpha}\left(\cdot, f^{n}\right)\right\}$. Then we have $P(f, \mathcal{F}(\alpha))=P^{*}(\alpha)$.

Proof. For a fixed $\alpha$, let $\phi_{n}(x)=-g^{\alpha}\left(x, f^{n}\right)$. Then it is a sub-additive continuous sequence on $\Lambda$. By Proposition 2.1 for $\mathcal{F}(\alpha)=\left\{-g^{\alpha}\left(x, f^{n}\right)\right\}$, we have

$$
P(f, \mathcal{F}(\alpha))=\lim _{n \rightarrow \infty} P\left(f, \frac{\phi_{n}}{n}\right)=\lim _{n \rightarrow \infty} P\left(f,-\frac{1}{n} g^{\alpha}\left(\cdot, f^{n}\right)\right)=P^{*}(\alpha) .
$$

Theorem 2.4. Let $\mathcal{F}(\alpha)=\left\{-g^{\alpha}\left(\cdot, f^{n}\right)\right\}$. Then we have that $P(f, \mathcal{F}(\alpha))$ is continuous and strictly monotone decreasing on $\alpha \in[0, m]$. Thus $P(f, \mathcal{F}(\alpha))=0$ has only a unique solution in $[0, m]$.

Proof. Let $\phi_{n}(\alpha, x)=-g^{\alpha}\left(x, f^{n}\right)$. If $\alpha_{1}, \alpha_{2} \in[0, m], \alpha_{1}<\alpha_{2}$, then for all $n \in \mathbb{N}$, we have

$$
\left(\alpha_{1}-\alpha_{2}\right) n \log k \geq-\phi_{n}\left(\alpha_{2}, x\right)-\left(-\phi_{n}\left(\alpha_{1}, x\right)\right) \geq\left(\alpha_{1}-\alpha_{2}\right) n \log \|f\| .
$$

Thus for $\forall \epsilon>0$,

$$
e^{\left(\alpha_{1}-\alpha_{2}\right) n \log k} \times P_{n}\left(\mathcal{F}\left(\alpha_{1}\right), \epsilon\right) \leq P_{n}\left(\mathcal{F}\left(\alpha_{2}\right), \epsilon\right) \leq P_{n}\left(\mathcal{F}\left(\alpha_{1}\right), \epsilon\right) \times e^{\left(\alpha_{1}-\alpha_{2}\right) n \log \|f\|} .
$$

This implies that

$$
\left(\alpha_{1}-\alpha_{2}\right) \log \|f\|+P\left(f, \mathcal{F}\left(\alpha_{1}\right)\right) \leq P\left(f, \mathcal{F}\left(\alpha_{2}\right)\right) \leq P\left(f, \mathcal{F}\left(\alpha_{1}\right)\right)+\left(\alpha_{1}-\alpha_{2}\right) \log k .
$$

Therefore $P(f, \mathcal{F}(\alpha))$ is continuous and strictly monotone decreasing on $\alpha \in[0, m]$.

On the one hand, $P(f, \mathcal{F}(0))=h_{\text {top }}(f)>0$, and on the other hand, by Ruelle's inequality [14] and Theorem 2.1, we have $P(f, \mathcal{F}(m)) \leq 0$. Therefore $P(f, \mathcal{F}(\alpha))=0$ has a unique solution in $[0, m]$.

Remark 2. Theorem 2.4 can be deduced from Theorem 2.3 and Lemma 2.1. But for the completeness, we include a different proof.

Corollary 2. $\mathcal{D}(\Lambda, f)$ is the unique solution of equation $P(f, \mathcal{F}(\alpha))=0$.

Proof. The proof can be deduced from Theorem 2.3 and Remark 1

Lemma 2.2. For a fixed $n \in \mathbb{N}, P_{n}(\alpha)=P\left(f,-\frac{1}{n} g^{\alpha}\left(\cdot, f^{n}\right)\right)$ is a continuous and monotone decreasing function on $\alpha \in[0, m]$.

Proof. The proof is analogous to the proof of Theorem 2.4.

By the Ruelle-Margulis inequality and the variational principle in [17, we have $P_{n}(m)=P\left(f,-\frac{1}{n} g^{m}\left(\cdot, f^{n}\right)\right) \leq 0$. Since $P_{n}(0)=h\left(\left.f\right|_{\Lambda}\right)>0$, by Lemma 2.2, it follows that equation $P_{n}(\alpha)=0$ has a unique solution. Denote it by $\alpha_{n}$. Then we have the following proposition.

\section{Theorem 2.5.}

$$
\inf _{n \in \mathbb{N}} \alpha_{n}=\mathcal{D}(\Lambda, f) .
$$

Proof. Without loss of generality, we suppose that $\lim _{n \rightarrow \infty} \alpha_{n}=\alpha^{*}=\inf _{n \in \mathbb{N}} \alpha_{n}$. Otherwise we can take a subsequence which converges to $\alpha^{*}$.

Since

$$
\begin{aligned}
\left|P\left(f,-\frac{1}{n} g^{\alpha^{*}}\left(\cdot, f^{n}\right)\right)-P\left(f,-\frac{1}{n} g^{\alpha_{n}}\left(\cdot, f^{n}\right)\right)\right| & \leq\left\|-\frac{1}{n} g^{\alpha^{*}}\left(\cdot, f^{n}\right)+\frac{1}{n} g^{\alpha_{n}}\left(\cdot, f^{n}\right)\right\| \\
& \leq\left|\alpha^{*}-\alpha_{n}\right|\|D f\|,
\end{aligned}
$$


we have

$$
\begin{aligned}
P\left(f, \mathcal{F}\left(\alpha^{*}\right)\right) & =\lim _{n \rightarrow \infty} P\left(f,-\frac{1}{n} g^{\alpha^{*}}\left(\cdot, f^{n}\right)\right) \\
& =\lim _{n \rightarrow \infty} P\left(f,-\frac{1}{n} g^{\alpha_{n}}\left(\cdot, f^{n}\right)\right)=0 .
\end{aligned}
$$

By Corollary 2, we have

$$
\mathcal{D}(f, \Lambda)=\alpha^{*}=\inf _{n \in \mathbb{N}} \alpha_{n}
$$

Now for a fixed $n \in \mathbb{N}$, we consider the equation

$$
\tilde{P}_{n}(\alpha)=P\left(\left.f^{n}\right|_{\Lambda},-g^{\alpha}\left(\cdot, f^{n}\right)\right)=0 .
$$

It is easy to prove that $\tilde{P}_{n}(\alpha)$ is continuous and strictly decreasing on $[0, m]$,

$$
\tilde{P}_{n}(0)=h_{\text {top }}\left(\left.f^{n}\right|_{\Lambda}\right)=n h_{\text {top }}\left(\left.f\right|_{\Lambda}\right) \geq 0
$$

and

$$
\tilde{P}_{n}(m)=n P\left(\left.f\right|_{\Lambda},-\log \left|J a c\left(D_{x} f\right)\right|\right) \leq 0 .
$$

Hence the equation $\tilde{P}_{n}(\alpha)=0$ has a unique solution, which we denoted by $D_{n}$. Applying Lemma 1 in [18] to the expanding map $f^{n}$ yields $\operatorname{dim}_{H} \Lambda \leq D_{n}$. So $\operatorname{dim}_{H} \Lambda \leq \inf _{n \in Z^{+}} D_{n}$. It was proved in [18] that

$$
\inf _{n \in Z^{+}} D_{n} \leq \mathcal{D}(f, \Lambda)
$$

Next we want to prove the reverse inequality, that is to say,

$$
\mathcal{D}(f, \Lambda) \leq \inf _{n \in Z^{+}} D_{n}
$$

In order to prove the inequality as above, we firstly prove the following theorem.

Proposition 2.2. Suppose $\left\{\phi_{n}(x)\right\}$ is a sub-additive continuous sequence on $M$. Let $\mathcal{F}=\left\{\phi_{n}\right\}$. Then we have $P(f, \mathcal{F})=\lim _{k \rightarrow \infty} \frac{1}{k} P\left(f^{k}, \phi_{k}\right)$.

Proof. For a fixed $k \in \mathbb{N}$, it is well known that if $E \subset M$ is an $(n, \epsilon)$-separated set of $f^{k}$, then $E$ is an $(n k, \epsilon)$-separated set of $f$. By the definition,

$$
P\left(f^{k}, \phi_{k}, \epsilon\right)=\lim _{\epsilon \rightarrow \infty} \limsup _{n \rightarrow \infty} \frac{1}{n} \log \sup \left\{\sum_{x \in E} \exp \left(\hat{S}_{n} \phi_{k}(x)\right) \mid\right.
$$

$E$ is an $(n, \epsilon)$-separated set of $\left.f^{k}\right\}$,

where

$$
\left(\hat{S}_{n} \phi_{k}(x)\right)=\phi_{k}(x)+\phi_{k}\left(f^{k} x\right)+\cdots+\phi_{k}\left(f^{(n-1) k} x\right) .
$$

Hence for a fixed $m<k$, letting $k=m q+r$ and $C=\max _{x \in M} \max _{i=1, \cdots, 2 m} \phi_{i}(x)$, the sub-additivity of $\phi_{n}$ implies that

$$
\begin{aligned}
\phi_{k}(x) & \leq \frac{1}{m} \sum_{j=0}^{m-1} \sum_{i=0}^{q-2} \phi_{m}\left(f^{i m+j}(x)\right)+\frac{1}{m} \sum_{j=0}^{m-1}\left[\phi_{j}(x)+\phi_{m-j+l}\left(f^{(q-1) m+j}(x)\right)\right] \\
& \leq \sum_{i=0}^{k-1} \frac{1}{m} \phi_{m}\left(f^{i}(x)\right)+4 C .
\end{aligned}
$$


Thus for $1 \leq j \leq n-1$, we have

$$
\phi_{k}\left(f^{k j}(x)\right) \leq \sum_{i=0}^{k-1} \frac{1}{m} \phi_{m}\left(f^{i}\left(f^{k j}(x)\right)+4 C .\right.
$$

Hence

$$
\begin{aligned}
\hat{S}_{n} \phi_{k}(x) & =\phi_{k}(x)+\phi_{k}\left(f^{k} x\right)+\cdots+\phi_{k}\left(f^{(n-1) k} x\right) \\
& \leq \sum_{i=0}^{n k-1} \frac{1}{m} \phi_{m}\left(f^{i}(x)\right)+4 n C \\
& =S_{n k}\left(\frac{1}{m} \phi_{m}\right)(x)+4 n C .
\end{aligned}
$$

This gives that

$$
P_{n}\left(f^{k}, \phi_{k}, \epsilon\right) \leq P_{n k}\left(f, \frac{1}{m} \phi_{m}, \epsilon\right) \times e^{4 n C} .
$$

Thus

$$
\begin{aligned}
P\left(f^{k}, \phi_{k}\right) & \leq k P\left(f, \frac{1}{m} \phi_{m}\right)+\lim _{n \rightarrow \infty} \frac{1}{n} \log e^{4 n C} \\
& =k P\left(f, \frac{1}{m} \phi_{m}\right)+4 C .
\end{aligned}
$$

Therefore

$$
\lim _{k \rightarrow \infty} \frac{1}{k} P\left(f^{k}, \phi_{k}\right) \leq P\left(f, \frac{1}{m} \phi_{m}\right) \quad \text { for all } m \in Z^{+} .
$$

By Theorem 2.1, we have

$$
\lim _{k \rightarrow \infty} \frac{1}{k} P\left(f^{k}, \phi_{k}\right) \leq \lim _{m \rightarrow \infty} P\left(f, \frac{1}{m} \phi_{m}\right)=P(f, \mathcal{F}) .
$$

Next we prove that

$$
P(f, \mathcal{F}) \leq \lim _{k \rightarrow \infty} \frac{1}{k} P\left(f^{k}, \phi_{k}\right) .
$$

For a fixed $k \in \mathbb{N}$, let $n=k m+r, 0 \leq r<k$, and let $C=\max _{x \in M} \max _{1 \leq i \leq k} \phi_{i}(x)$. For $\forall \epsilon>0$, by the uniform continuity of $f$, there exists $\delta>0$ such that if $E \subset M$ is an $(n, \epsilon)$-separated set of $f$, then $E$ is an $(m, \delta)$-separated set of $f^{k}$ and $\delta \rightarrow 0$ when $\epsilon \rightarrow 0$. Using the sub-additivity of $\phi_{n}$, we have

$$
\phi_{n}(x) \leq \phi_{k}(x)+\phi_{k}\left(f^{k}(x)\right)+\cdots+\phi_{k}\left(f^{(m-1) k}(x)\right)+\phi_{r}\left(f^{m k}(x)\right) .
$$

Thus

$$
P_{n}(f, \mathcal{F}, \epsilon) \leq P_{m}\left(f^{k}, \phi_{k}, \delta\right) \times e^{C} .
$$

Hence

$$
P(f, \mathcal{F}, \epsilon) \leq \frac{1}{k} P\left(f^{k}, \phi_{k}, \delta\right) .
$$

This gives that

$$
P(f, \mathcal{F}) \leq \frac{1}{k} P\left(f^{k}, \phi_{k}\right) .
$$

For $k$ arbitrary, we have that

$$
P(f, \mathcal{F}) \leq \lim _{k \rightarrow \infty} \frac{1}{k} P\left(f^{k}, \phi_{k}\right) .
$$


Corollary 3. Let $\mathcal{F}(\alpha)=\left\{-g^{\alpha}\left(\cdot, f^{k}\right)\right\}$. Then we have

$$
P(f, \mathcal{F}(\alpha))=\lim _{k \rightarrow \infty} \frac{1}{k} P\left(f^{k},-g^{\alpha}\left(\cdot, f^{k}\right)\right) .
$$

Proof. For fixed $\alpha$, let $\phi_{k}(x)=-g^{\alpha}\left(x, f^{k}\right)$. Using Theorem 2.2 for $\mathcal{F}(\alpha)=$ $\left\{-g^{\alpha}\left(x, f^{k}\right)\right\}$, we get

$$
P(f, \mathcal{F}(\alpha))=\lim _{k \rightarrow \infty} \frac{1}{k} P\left(f^{k},-g^{\alpha}\left(\cdot, f^{k}\right)\right) .
$$

Theorem 2.6.

$$
\inf _{n \in \mathbb{N}} D_{n}=\mathcal{D}(\Lambda, f) .
$$

Proof. Without loss of generality, we suppose that $\lim _{n \rightarrow \infty} D_{n}=\beta^{*}=\inf _{n \in \mathbb{N}} D_{n}$. Otherwise we can take a subsequence which converges to $\beta^{*}$. Since

$$
\begin{aligned}
\left|\frac{1}{k} P\left(f^{k},-g^{\beta^{*}}\left(\cdot, f^{k}\right)\right)-\frac{1}{k} P\left(f^{k},-g^{D_{k}}\left(\cdot, f^{k}\right)\right)\right| & \leq\left\|-\frac{1}{k} g^{\beta^{*}}\left(\cdot, f^{k}\right)+\frac{1}{k} g^{D_{k}}\left(\cdot, f^{k}\right)\right\| \\
& \leq\left|\beta^{*}-D_{k}\right|\|D f\|,
\end{aligned}
$$

we have

$$
\begin{aligned}
P\left(f, \mathcal{F}\left(\beta^{*}\right)\right) & =\lim _{k \rightarrow \infty} \frac{1}{k} P\left(f^{k},-g^{\beta^{*}}\left(\cdot, f^{k}\right)\right) \\
& =\lim _{k \rightarrow \infty} \frac{1}{k} P\left(f^{k},-g^{D_{k}}\left(\cdot, f^{k}\right)\right)=0 .
\end{aligned}
$$

Thus

$$
\mathcal{D}(f, \Lambda)=\beta^{*}=\inf _{k \in \mathbb{N}} D_{k}
$$

In this section, we have proven that for a $C^{1}$ non-conformal repeller $\Lambda, \mathcal{D}(f, \Lambda)$, which is the unique solution of equation $P(f, \mathcal{F}(\alpha))=0$, is the upper bound of the Hausdorff dimension of $\Lambda$. This is a generalization of the classical result that for a $C^{1+\gamma}$ conformal repeller $\Lambda, \operatorname{dim}_{H} \Lambda$ is given by the unique solution of the equation $P\left(\left.f\right|_{\Lambda},-\alpha \log \left\|D_{x} f\right\|\right)=0$. Moreover, we prove that

$$
\mathcal{D}(f, \Lambda)=\inf _{n \in \mathbb{N}} D_{k}=\inf _{n \in \mathbb{N}} \alpha_{k}
$$

where for each $n \in \mathbb{N}, D_{n}$ and $\alpha_{n}$ are the unique solutions of the equations $\tilde{P}_{n}(\alpha)=$ 0 and $P_{n}(\alpha)=0$, respectively.

\section{OtheR RESUlts OF UPPER BOUND ESTIMATES OF THE Dimension FOR A REPELLER}

Let us first recall Falconer's definition of topological pressures for sub-additive potentials on mixing repellers. Without loss of generality, we only consider onesided sub-shift spaces of finite type rather than mixing repellers.

Let $\left(\Sigma_{A}, \sigma\right)$ be a one-sided sub-shift space over an alphabet $\{1, \ldots, m\}$, where $m \geq 2$. As usual $\Sigma_{A}$ is endowed with the metric $d(x, y)=m^{-n}$, where $x=$ $\left(x_{k}\right), y=\left(y_{k}\right)$ and $n$ is the smallest of the $k$ such that $x_{k} \neq y_{k}$. For any admissible string $I=i_{1} \ldots i_{n}$ of length $n$ over the letters $\{1, \ldots, m\}$, denote $[I]=\left\{\left(x_{i}\right) \in \Sigma\right.$ : $x_{j}=i_{j}$ for $\left.1 \leq j \leq n\right\}$. The $[I]$ is called an $n$-th cylinder in $\Sigma_{A}$. 
Let $\mathcal{F}$ be a sub-additive family of continuous potentials defined on $\Sigma$. Falconer defined the topological pressure of $\mathcal{F}$ by

$$
F P(\sigma, \mathcal{F})=\lim _{n \rightarrow \infty} \frac{1}{n} \log F P_{n}(\sigma, \mathcal{F}) \quad \text { and } \quad F P_{n}(\sigma, \mathcal{F})=\sum_{[I]} \sup _{x \in[I]} e^{\phi_{n}(x)},
$$

where the summation is taken over the collection of all $n$th cylinders $[I]$.

It is not so hard to see that in this special case, $F P_{n}(\sigma, \mathcal{F})=P_{n}(\sigma, \mathcal{F}, 1 / m)$ and $P_{n}\left(\sigma, \mathcal{F}, m^{-k}\right)=F P_{n+k-1}(\sigma, \mathcal{F})$ for all $k \in \mathbb{N}$. This implies that $F P(\sigma, \mathcal{F})$ is equivalent to our definition of $P(\sigma, \mathcal{F})$.

Now let us turn to Barreira's approach in defining pressures for sub-additive potentials via open covers.

As in the previous sections, let $f$ be a continuous map acting on a compact metric space $(X, d)$. Let $\mathcal{F}=\left\{\phi_{n}\right\}_{n=1}^{\infty}$ be a family of sub-additive continuous functions defined on $X$. Suppose $\mathcal{U}$ is a finite open cover of the space $X$. For $n \geq 1$ we denote by $\mathcal{W}_{n}(\mathcal{U})$ the collection of strings $\mathbf{U}=U_{1} \ldots U_{n}$ with $U_{i} \in \mathcal{U}$. For $\mathbf{U} \in \mathcal{W}_{n}(\mathcal{U})$ we call the integer $m(\mathbf{U})=n$ the length of $\mathbf{U}$ and define

$$
\begin{aligned}
X(\mathbf{U}) & =U_{1} \cap f^{-1} U_{2} \cap \ldots \cap f^{-(n-1)} U_{n} \\
& =\left\{x \in X: f^{j-1} x \in U_{j} \text { for } j=1, \ldots, n\right\} .
\end{aligned}
$$

We say that $\Gamma \subset \bigcup_{n>1} \mathcal{W}_{n}(\mathcal{U})$ covers $X$ if $\bigcup_{\mathbf{U} \in \Gamma} X(\mathbf{U})=X$. For each $\mathbf{U} \in \mathcal{W}_{n}(\mathcal{U})$, we write $e^{\phi(\mathbf{U})}=\sup _{x \in X(\mathbf{U})} e^{\phi_{n}(x)}$ when $X(\mathbf{U}) \neq \emptyset$ and $e^{\phi(\mathbf{U})}=-\infty$ otherwise. For $s \in \mathbb{R}$, define

$$
M(f, s, \mathcal{F}, \mathcal{U})=\lim _{n \rightarrow \infty} \inf \left\{\sum_{\mathbf{U} \in \Gamma} e^{-s m(\mathbf{U})} e^{\phi(\mathbf{U})}\right\},
$$

where the infimum is taken over all $\Gamma \subset \bigcup_{j \geq n} \mathcal{W}_{j}(\mathcal{U})$ that cover $X$. Likewise, we define

$$
\begin{aligned}
& \underline{M}(f, s, \mathcal{F}, \mathcal{U})=\liminf _{n \rightarrow \infty} \inf \left\{\sum_{\mathbf{U} \in \Gamma} e^{-s m(\mathbf{U})} e^{\phi(\mathbf{U})}\right\}, \\
& \bar{M}(f, s, \mathcal{F}, \mathcal{U})=\limsup _{n \rightarrow \infty} \inf \left\{\sum_{\mathbf{U} \in \Gamma} e^{-s m(\mathbf{U})} e^{\phi(\mathbf{U})}\right\},
\end{aligned}
$$

where the infimum is taken over all $\Gamma \subset \mathcal{W}_{n}(\mathcal{U})$ that cover $X$. Define

$$
\begin{gathered}
P^{\star}(f, \mathcal{F}, \mathcal{U})=\inf \{s: M(f, s, \mathcal{F}, \mathcal{U})=0\}=\sup \{s: M(f, s, \mathcal{F}, \mathcal{U})=+\infty\}, \\
\underline{C P^{\star}}(f, \mathcal{F}, \mathcal{U})=\inf \{s: \underline{M}(f, s, \mathcal{F}, \mathcal{U})=0\}=\sup \{s: \underline{M}(f, s, \mathcal{F}, \mathcal{U})=+\infty\}, \\
\overline{C P^{\star}}(f, \mathcal{F}, \mathcal{U})=\inf \{s: \bar{M}(f, s, \mathcal{F}, \mathcal{U})=0\}=\sup \{s: \bar{M}(f, s, \mathcal{F}, \mathcal{U})=+\infty\} .
\end{gathered}
$$

Define

$$
\begin{aligned}
P^{\star}(f, \mathcal{F}) & =\liminf _{\operatorname{diam}(\mathcal{U}) \rightarrow 0} P^{\star}(f, \mathcal{F}, \mathcal{U}), \\
\underline{C P^{\star}}(f, \mathcal{F}) & =\liminf _{\operatorname{diam}(\mathcal{U}) \rightarrow 0} \underline{C P^{\star}}(f, \mathcal{F}, \mathcal{U}), \\
\overline{C P^{\star}}(f, \mathcal{F}) & =\liminf _{\operatorname{diam}(\mathcal{U}) \rightarrow 0} \overline{C P^{\star}}(f, \mathcal{F}, \mathcal{U}) .
\end{aligned}
$$

Barreira named $P^{\star}(f, \mathcal{F})$ the topological pressure, $\underline{C P^{\star}}(f, \mathcal{F})$ and $\overline{C P^{\star}}(f, \mathcal{F})$ the lower and upper topological pressures of $\mathcal{F}$.

Now we consider the connection between $P^{\star}(f, \mathcal{F})$ and $P(f, \mathcal{F})$. In [4], we prove the following equality. 
Proposition 3.1. Assume that the topological entropy $h(f)<\infty$ and that the entropy map $\mu \mapsto h_{\mu}(f)$ is upper-semi-continuous. Then $P^{\star}(f, \mathcal{F})=P(f, \mathcal{F})$.

Theorem 3.1. Let $M$ be a $C^{\infty}$ Riemann manifold and $f: M \rightarrow M$ be a $C^{1}$ map. Suppose $\Lambda \subset M$ is a compact invariant set on which $f$ is expanding. Then

$$
P^{\star}(f, \mathcal{F}(\alpha))=P(f, \mathcal{F}(\alpha)) .
$$

Proof. Since $\Lambda \subset M$ is a compact invariant set on which $f$ is expanding, it has measure-theoretic entropy and $h_{\mu}\left(\left.f\right|_{\Lambda}\right)$ is an upper-semi-continuous map in $\mathcal{M}\left(\left.f\right|_{\Lambda}\right)$. By Proposition 3.1, we have

$$
P^{\star}(f, \mathcal{F}(\alpha))=P(f, \mathcal{F}(\alpha)) .
$$

In 1, Barreira proved that if $\Lambda$ is a repeller of a $C^{1+\gamma}$ map, for some $\gamma>0$ and $f$ is $\gamma$-bunched on $\Lambda$, then $\overline{\operatorname{dim}}_{B} \leq t^{*}$, where $t^{*}$ is the unique number of equations $P^{\star}(f, \mathcal{F}(\alpha))=0$. In [1], the $\gamma$-bunched condition and $C^{1+\gamma}$ were used to show that it is reasonable to define $P^{\star}(f, \mathcal{F}(\alpha))$.

Corollary 4. Let $M$ be a $C^{\infty}$ Riemann manifold and $f: M \rightarrow M$ be a $C^{1}$ map. Suppose $\Lambda \subset M$ is a compact invariant set on which $f$ is expanding. Then

$$
\operatorname{dim}_{B} \Lambda \leq \mathcal{D}(\Lambda, f) \text { and } \operatorname{dim}_{H} \Lambda \leq \mathcal{D}(\Lambda, f),
$$

where $\mathcal{D}(\Lambda, f)$ is the unique solution of the equation $P(f, \mathcal{F}(\alpha))=0$.

Proof. By Theorem 3.1, we have that if $\Lambda$ is a repeller of a $C^{1}$ map, then we can define $P^{\star}(f, \mathcal{F}(\alpha))$ and prove that it is coincident with $P(f, \mathcal{F}(\alpha))$. It is proved in [1] that $\overline{\operatorname{dim}}_{B} \leq t^{*}$, where $t^{*}$ is the unique solution of the equation $P^{\star}(f, \mathcal{F}(\alpha))=0$. Thus we have that $t^{*}=\mathcal{D}(\Lambda, f)$, which is the unique solution of the equation $P(f, \mathcal{F}(\alpha))=0$. Therefore we also have the inequality for the box dimension.

Remark 3. In [18, Zhang posed a problem whether $\mathcal{D}(\Lambda, f)$ is the upper bound of the box dimension of $\Lambda$. The corollary as above gives an affirmative answer to the problem. Moreover, our result shows that the sub-additive thermodynamic formalism can be applied. In fact we have proven that if $\Lambda$ is a repeller of a $C^{1}$ map, then the upper bounds of the Hausdorff dimension of $\Lambda$ by Barreira in [1, Falconer in [8] and Zhang in [18] all coincide. This unifies their results and it also shows that the bunched condition in [1] and [8] is unnecessary. Our result also gives an affirmative answer to a problem posed by K. Simon in [15] concerning an upper bound without assuming the 1-bunched property.

\section{Average conformal repeller}

Let $M$ be a $C^{\infty}$ Riemann manifold, $\operatorname{dim} M=d$. Let $U$ be an open subset of $M$ and let $f: U \rightarrow M$ be a $C^{1}$ map. Suppose $\Lambda \subset U$ is a compact expanding invariant set. Let $\mathcal{E}(f)$ denote all the ergodic invariant measures supported on $\Lambda$. By the Oseledec multiplicative ergodic theorem, for any $\mu \in \mathcal{E}(f)$, we can define Lyapunov exponents $\lambda_{1}(\mu) \leq \lambda_{2}(\mu) \leq \cdots \leq \lambda_{d}(\mu)$.

Definition 4.1. An invariant repeller is called average conformal if for any $\mu \in$ $\mathcal{E}(f), \lambda_{1}(\mu)=\lambda_{2}(\mu)=\cdots=\lambda_{d}(\mu)>0$. 
It is obvious that a conformal repeller is an average conformal repeller, but the reverse is not true.

Next we will give the main theorem.

Theorem 4.1 (Main Theorem). Let $f$ be a $C^{1}$ dynamical system and let $\Lambda$ be an average conformal repeller. Then the Hausdorff dimension of $\Lambda$ is the zero $t_{0}$ of $t \mapsto P(-t \mathcal{F})$, where

$$
\mathcal{F}=\left\{\log \left(m\left(D f^{n}(x)\right)\right), x \in \Lambda, n \in \mathbb{N}\right\},
$$

and where $m(A)=\left\|A^{-1}\right\|^{-1}$

The proof will be given in Section 6 .

Theorem 4.2. If $\Lambda$ is an average conformal repeller, then

$$
\lim _{n \rightarrow \infty} \frac{1}{n}\left(\log \left\|D f^{n}(x)\right\|-\log m\left(D f^{n}(x)\right)\right)=0
$$

uniformly on $\Lambda$.

Proof. Let

$$
F_{n}(x)=\log \left\|D f^{n}(x)\right\|-\log m\left(D f^{n}(x)\right), n \in \mathbb{N}, x \in \Lambda .
$$

It is obvious that the sequence $\left\{F_{n}(x)\right\}$ is a non-negative sub-additive function sequence. That is to say,

$$
F_{n+m}(x) \leq F_{n}(x)+F_{m}\left(f^{n}(x)\right), x \in \Lambda .
$$

Suppose (4.3) is not true. Then there exists $\epsilon_{0}>0$, and for any $k \in \mathbb{N}$, there exists $n_{k} \geq k$ and $x_{n_{k}} \in \Lambda$ such that

$$
\frac{1}{n_{k}} F_{n_{k}}\left(x_{n_{k}}\right) \geq \epsilon_{0}
$$

Define measures

$$
\mu_{n_{k}}=\frac{1}{n_{k}} \sum_{i=0}^{n_{k}-1} \delta_{f^{i}\left(x_{n_{k}}\right)} .
$$

The compactness of $\mathcal{P}(f)$ implies that there exists a subsequence of $\mu_{n_{k}}$ that converges to the measure $\mu$. Without loss of generality, we suppose that $\mu_{n_{k}} \rightarrow \mu$. It is well known that $\mu$ is $f$-invariant. Therefore $\mu \in \mathcal{M}(f)$.

For a fixed $m$, we have

$$
\lim _{k \rightarrow \infty} \int_{M} \frac{1}{m} F_{m}(x) d \mu_{n_{k}}=\int_{M} \frac{1}{m} F_{m}(x) d \mu .
$$

This implies

$$
\lim _{k \rightarrow \infty} \frac{1}{n_{k}} \sum_{i=0}^{n_{k}-1} \frac{1}{m} F_{m}\left(f^{i}\left(x_{n_{k}}\right)\right)=\int_{M} \frac{1}{m} F_{m}(x) d \mu .
$$

For a fixed $m$, let $n_{k}=m s+l, 0 \leq l<m$. The sub-additivity of $\left\{F_{n}\right\}$ implies that for $j=0, \ldots, m-1$,

$$
\begin{aligned}
F_{n_{k}}\left(x_{n_{k}}\right) \leq & F_{j}\left(x_{n_{k}}\right)+F_{m}\left(f^{j}\left(x_{n_{k}}\right)+\cdots+F_{m}\left(f^{m(s-2)} f^{j}\left(x_{n_{k}}\right)\right)\right. \\
& +F_{m-j+l}\left(f^{m(s-1)} f^{j}\left(x_{n_{k}}\right)\right) .
\end{aligned}
$$


Summing $j$ from 0 to $m-1$, we get

$$
\begin{aligned}
F_{n_{k}}\left(x_{n_{k}}\right) & \leq \frac{1}{m} \sum_{j=0}^{m-1} \sum_{i=0}^{s-2} F_{m}\left(f^{i m+j}\left(x_{n_{k}}\right)\right) \\
& +\frac{1}{m} \sum_{j=0}^{m-1}\left[F_{j}\left(x_{n_{k}}\right)+F_{m-j+l}\left(f^{(s-1) m+j}\left(x_{n_{k}}\right)\right)\right] .
\end{aligned}
$$

Let $C_{1}=\max _{i=1, \ldots, 2 m-1} \max _{x \in \Lambda} F_{i}(x)$. Then

$$
\begin{aligned}
F_{n_{k}}(x) & \leq \sum_{j=0}^{(s m+l)-1} \frac{1}{m} F_{m}\left(f^{j}(x)\right)-\frac{1}{m} \sum_{j=(s-1) m}^{s m-1} F_{m}\left(f^{j}(x)\right)+2 C_{1} \\
& \leq \sum_{j=0}^{n_{k}-1} \frac{1}{m} F_{m}\left(f^{j}(x)\right)+4 C_{1} .
\end{aligned}
$$

Hence we have

$$
\lim _{k \rightarrow \infty} \frac{1}{n_{k}} F_{n_{k}}\left(x_{n_{k}}\right) \leq \lim _{k \rightarrow \infty} \frac{1}{n_{k}} \sum_{i=0}^{n_{k}-1} \frac{1}{m} F_{m}\left(f^{i}\left(x_{n_{k}}\right)\right)=\int_{M} \frac{1}{m} F_{m}(x) d \mu .
$$

The arbitrariness of $m \in \mathbb{N}$ implies that

$$
\lim _{k \rightarrow \infty} \frac{1}{n_{k}} F_{n_{k}}\left(x_{n_{k}}\right) \leq \frac{1}{m} \int_{M} F_{m}(x) d \mu, \forall m \in \mathbb{N} .
$$

Hence

$$
\lim _{m \rightarrow \infty} \frac{1}{m} \int_{M} F_{m}(x) d \mu \geq \epsilon_{0}>0 .
$$

Then the ergodic decomposition theorem [17] implies that there exists $\tilde{\mu} \in \mathcal{E}(f)$ such that

$$
\lim _{m \rightarrow \infty} \frac{1}{m} \int_{M} F_{m}(x) d \tilde{\mu} \geq \epsilon_{0}>0 .
$$

On the other hand, from the Oseledec theorem and Kingman's sub-additive ergodic theorem, we have

$$
\lim _{m \rightarrow \infty} \frac{1}{m} \int_{M} \log \left\|D f^{m}(x)\right\| d \tilde{\mu}=\lambda_{d}(\tilde{\mu}) \text { and } \lim _{m \rightarrow \infty} \frac{1}{m} \int_{M} \log m\left(D f^{m}(x)\right) d \tilde{\mu}=\lambda_{1}(\tilde{\mu}) .
$$

Therefore

$$
\lambda_{d}(\tilde{\mu})-\lambda_{1}(\tilde{\mu}) \geq \epsilon_{0} .
$$

This gives a contradiction to the assumption of an average conformal repeller.

\section{SuPER-ADDitive VARIATIONAL PRINCIPLE}

In this section, we first give the definition of super-additive topological pressure. Then we prove the variational principle for a special super-additive potential.

Let $f: X \rightarrow X$ be a continuous map. A set $E \subset X$ is called an $(n, \epsilon)$-separated set with respect to $f$ if whenever $x, y \in E$, then $d_{n}(x, y)=\max _{0 \leq i \leq n-1} d\left(f^{i} x, f^{i} y\right)$ $>\epsilon$. A super-additive valuation on $X$ is a sequence of functions $\varphi_{n}: M \rightarrow R$ such 
that

$$
\varphi_{m+n}(x) \geq \varphi_{n}(x)+\varphi_{m}\left(f^{n}(x)\right)
$$

we denote it by $\mathcal{F}=\left\{\varphi_{n}\right\}$.

In the following we will define the topological pressure of $\mathcal{F}=\left\{\varphi_{n}\right\}$ with respect to $f$. We define

$$
P_{n}^{*}(\mathcal{F}, \epsilon)=\sup \left\{\sum_{x \in E} \exp \varphi_{n}(x): E \quad \text { is an }(n, \epsilon) \text {-separated subset of } X\right\} .
$$

Then the topological pressure of $\mathcal{F}$ is given by

$$
P^{*}(f, \mathcal{F})=\lim _{\epsilon \rightarrow 0} \limsup _{n \rightarrow \infty} \frac{1}{n} \log P_{n}(\mathcal{F}, \epsilon) .
$$

For every $\mu \in \mathcal{M}(X, f)$, let $\mathcal{F}_{*}(\mu)$ denote the following limit:

$$
\mathcal{F}_{*}(\mu)=\lim _{n \rightarrow \infty} \frac{1}{n} \int \varphi_{n} d \mu .
$$

The existence of the above limit follows from a super-additive argument. We call $\mathcal{F}_{*}(\mu)$ the Lyapunov exponent of $\mathcal{F}$ with respect to $\mu$ since it describes the exponential growth speed of $\varphi_{n}$ with respect to $\mu$.

Theorem 5.1. Let $f$ be a $C^{1}$ dynamical system and let $\Lambda$ be an average conformal repeller. Let $\mathcal{F}=\left\{\varphi_{n}(x)\right\}=\left\{-t \log \left\|D f^{n}(x)\right\|\right\}$ for $t \geq 0$ be a super-additive function sequence. Then we have

$$
P^{*}(f, \mathcal{F})=\sup \left\{h_{\mu}(T)+\mathcal{F}_{*}(\mu): \mu \in \mathcal{M}(X, f)\right\} .
$$

Proof. First we prove that for any $m \in \mathbb{N}$,

$$
P^{*}(f, \mathcal{F}) \geq P\left(f, \frac{\varphi_{m}}{m}\right) .
$$

For a fixed $m$, let $n=m s+l, 0 \leq l<m$. From the super-additivity of $\left\{\varphi_{n}\right\}$, we have

$$
\varphi_{n}(x) \geq \frac{1}{m} \sum_{j=0}^{m-1} \sum_{i=0}^{s-2} \varphi_{m}\left(f^{i m+j}(x)\right)+\frac{1}{m} \sum_{j=0}^{m-1}\left[\varphi_{j}(x)+\varphi_{m-j+l}\left(f^{(s-1) m+j}(x)\right)\right] .
$$

Let $C_{1}=\min _{i=1, \ldots, 2 m-1} \min _{x \in X} \varphi_{i}(x)$. Then we have

$$
\begin{aligned}
\varphi_{n}(x) & \geq \sum_{j=0}^{(s m+l)-1} \frac{1}{m} \varphi_{m}\left(f^{j}(x)\right)-\frac{1}{m} \sum_{j=(s-1) m}^{s m-1} \varphi_{m}\left(f^{j}(x)\right)+2 C_{1} \\
& \geq \sum_{j=0}^{n-1} \frac{1}{m} \varphi_{m}\left(f^{j}(x)\right)+4 C_{1} .
\end{aligned}
$$

Hence we have

$$
\exp \left(\varphi_{n}(x)\right) \geq \exp \left(\sum_{j=0}^{n-1} \frac{1}{m} \varphi_{m}\left(f^{j}(x)\right)+4 C_{1}\right)
$$

Thus

$$
\begin{aligned}
P_{n}^{*}(\mathcal{F}, \epsilon) & =\sup \left\{\sum_{x \in E} \exp \varphi_{n}(x): E \text { is an }(n, \epsilon) \text {-separated subset of } X\right\} \\
& \geq P_{n}\left(\frac{1}{m} \varphi_{m}, \epsilon\right) \times \exp \left(4 C_{1}\right) .
\end{aligned}
$$


This implies

$$
P^{*}(f, \mathcal{F}) \geq P\left(f, \frac{1}{m} \varphi_{m}\right) .
$$

For an arbitrary $m \in Z^{+}$, we have

$$
P^{*}(f, \mathcal{F}) \geq P\left(f, \frac{1}{m} \varphi_{m}\right), \text { for all } m \in Z^{+} .
$$

By the variational principle in [17, for every $\mu \in \mathcal{M}(f)$, we have

$$
P^{*}(f, \mathcal{F}) \geq P\left(f, \frac{1}{m} \varphi_{m}\right) \geq h_{\mu}(f)+\int_{M} \frac{1}{m} \varphi_{m}(x) d \mu, \forall m \in \mathbb{N} .
$$

Hence we have for every $\mu \in \mathcal{M}(f)$,

$$
P^{*}(f, \mathcal{F}) \geq h_{\mu}(f)+\lim _{m \rightarrow \infty} \int_{M} \frac{1}{m} \varphi_{m}(x) d \mu .
$$

Therefore

$$
P^{*}(f, \mathcal{F}) \geq \sup \left\{h_{\mu}(f)+\lim _{m \rightarrow \infty} \int_{M} \frac{1}{m} \varphi_{m}(x) d \mu, \quad \mu \in \mathcal{M}(f)\right\} .
$$

Let $\Phi_{n}(x)=-t \log m\left(D f^{n}(x)\right)$ for $t \geq 0$. Then it is sub-additive. By the theorem in 4], we have

$$
P\left(f,\left\{\Phi_{n}\right\}\right)=\sup \left\{h_{\mu}(f)+\lim _{m \rightarrow \infty} \int_{M} \frac{1}{m} \Phi_{m}(x) d \mu, \mu \in \mathcal{M}(f)\right\} .
$$

By the definitions, $-t \log m\left(D f^{n}(x)\right) \geq-t \log \left\|D f^{n}(x)\right\|$ for $t \geq 0$ implies that

$$
P^{*}(f, \mathcal{F}) \leq P\left(f,\left\{\Phi_{n}\right\}\right) .
$$

Theorem 4.3 implies that for any $\mu \in \mathcal{M}(f)$, we have

$$
\lim _{m \rightarrow \infty} \int_{M} \frac{1}{m} \Phi_{m}(x) d \mu=\lim _{m \rightarrow \infty} \int_{M} \frac{1}{m} \varphi_{m}(x) d \mu .
$$

Therefore

$$
P^{*}(f, \mathcal{F})=\sup \left\{h_{\mu}(f)+\lim _{m \rightarrow \infty} \int_{M} \frac{1}{m} \Phi_{m}(x) d \mu, \quad \mu \in \mathcal{M}(f)\right\} .
$$

This completes the proof of the theorem.

\section{The PRoOF OF THE MAIN THEOREM}

In this section, we will give the proof of the main theorem. First we state some known results.

In [1], Barreira proved the following theorem.

Theorem 6.1. If $f$ is a $C^{1}$ expanding map and $\Lambda$ is a repeller, then

$$
s_{1} \leq \operatorname{dim}_{H} \Lambda \leq \underline{\operatorname{dim}}_{B} \Lambda \leq \overline{\operatorname{dim}}_{B} \Lambda \leq t_{1},
$$

where $s_{1}$ and $t_{1}$ are the unique roots of Bowen's equations $P(f,-t \log \|D f(x)\|)=0$ and $P(f,-t \log m(D f(x)))=0$, respectively.

Since $\Lambda$ is $f$-invariant, it is $f^{n}$-invariant. Hence we have the following corollary.

Corollary 5. If $f$ is a $C^{1}$ expanding map and $\Lambda$ is a repeller, then

$$
s_{n} \leq \operatorname{dim}_{H} \Lambda \leq \underline{\operatorname{dim}}_{B} \Lambda \leq \overline{\operatorname{dim}}_{B} \Lambda \leq t_{n},
$$

where $s_{n}$ and $t_{n}$ are the unique roots of Bowen's equations $P\left(f^{n},-t \log \left\|D f^{n}(x)\right\|\right)$ $=0$ and $P\left(f^{n},-t \log m\left(D f^{n}(x)\right)\right)=0$, respectively. 
Next we prove that the sequences $\left\{t_{2^{k}}\right\}$ and $\left\{s_{2^{k}}\right\}$ are monotone.

Theorem 6.2. The sequence $\left\{s_{2^{k}}\right\}$ is monotone, and

$$
\lim _{k \rightarrow \infty} s_{2^{k}}=s_{*} .
$$

Then we have that $s_{*}$ is the unique root of the equation $P^{*}\left(f,-t\left\{\log \left\|D f^{n}(x)\right\|\right\}\right)$ $=0$.

Proof. First we prove that the sequence $\left\{s_{2^{n}}\right\}$ is monotone increasing. Let $\varphi_{n}=$ $-\log \|\left(D f^{n}(x) \|\right.$ and $\mathcal{F}=\left\{\varphi_{n}\right\}$. Then it is a super-additive function sequence. For a fixed $k \in \mathbb{N}$,

$$
P_{k}(\phi, \epsilon)=\sup \left\{\sum_{x \in E} \exp S_{n} \phi(x): E \text { is an }(n, \epsilon) \text {-separated subset of } X\right\} .
$$

For $\forall \epsilon>0$, by the uniform continuity of $f$, there exists $\delta>0$ such that if $E \subset M$ is an $(n, \epsilon)$-separated set of $f^{2^{k+1}}$, then $E$ is a $(2 n, \delta)$-separated set of $f^{2^{k}}$ and $\delta \rightarrow 0$ when $\epsilon \rightarrow 0$. Using the super-additivity of $\varphi_{n}$, the Birkhoff sum $S_{n} \varphi_{2^{k+1}}$ of $\varphi_{2^{k+1}}$ with respect to $f^{2^{k+1}}$ has the following property:

$$
\begin{aligned}
S_{n} \varphi_{2^{k+1}}(x) & =\varphi_{2^{k+1}}(x)+\varphi_{2^{k+1}}\left(f^{2^{k+1}} x\right)+\cdots+\varphi_{2^{k+1}}\left(f^{2^{k+1}(n-1)} x\right) \\
& \geq \varphi_{2^{k}}(x)+\varphi_{2^{k}}\left(f^{2^{k}} x\right)+\varphi_{2^{k}}\left(f^{2^{k+1}} x\right)+\varphi_{2^{k}}\left(f^{2^{k+1}} f^{2^{k}} x\right) \\
& +\cdots+\varphi_{2^{k}}\left(f^{2^{k+1}(n-1)} x\right)+\varphi_{2^{k}}\left(f^{2^{k+1}(n-1)} f^{2^{k}} x\right) \\
& =S_{2 n} \varphi_{2^{k}}(x),
\end{aligned}
$$

where $S_{2 n} \varphi_{2^{k}}(x)$ is the Birkhoff sum of $\varphi_{2^{k}}$ with respect to $f^{2^{k}}$.

Thus

$$
P_{n}\left(f^{2^{k+1}}, \varphi_{2^{k+1}}, \epsilon\right) \geq P_{2 n}\left(f^{2^{k}}, \varphi_{2^{k}}, \delta\right)
$$

Hence

$$
P\left(f^{2^{k+1}}, \varphi_{2^{k+1}}\right) \geq 2 P\left(f^{2^{k}}, \varphi_{2^{k}}\right) .
$$

Therefore if $s_{2^{k+1}}$ is the unique root of Bowen's equation $P\left(t \varphi_{2^{k+1}}\right)=0$, then we have

$$
0=P\left(f^{2^{k+1}}, s_{2^{k+1}} \varphi_{2^{k+1}}\right) \geq 2 P\left(f^{2^{k}}, s_{2^{k+1}} \varphi_{2^{k}}\right) .
$$

Since the function $P\left(f^{2^{k}}, t \varphi_{2^{k}}\right)$ is monotone decreasing, $s_{2^{k}} \leq s_{2^{k+1}}$.

The arbitrariness of $k$ implies that the sequence $\left\{s_{2^{k}}\right\}$ is monotone decreasing.

Next we prove that

$$
P^{*}(f, \mathcal{F}) \geq \frac{1}{k} P\left(f^{k}, \varphi_{k}\right) \quad \forall k \in \mathbb{N} .
$$

For a fixed $k \in \mathbb{N}$, let $n=k m+r, 0 \leq r<k$, and let $C=\min _{x \in M} \max _{1 \leq i \leq k} \varphi_{i}(x)$. For $\forall \epsilon>0$, by the uniformly continuity of $f$, there exists $\delta>0$ such that if $E \subset M$ is an $(n, \epsilon)$-separated set of $f$, then $E$ is an $(m, \delta)$-separated set of $f^{k}$ and $\delta \rightarrow 0$ when $\epsilon \rightarrow 0$. Using the super-additivity of $\varphi_{n}$, we have

$$
\varphi_{n}(x) \geq \varphi_{k}(x)+\varphi_{k}\left(f^{k}(x)\right)+\cdots+\varphi_{k}\left(f^{(m-1) k}(x)\right)+\varphi_{r}\left(f^{m k}(x)\right) .
$$

Thus

$$
P_{n}^{*}(f, \mathcal{F}, \epsilon) \geq P_{m}\left(f^{k}, \varphi_{k}, \delta\right) \times e^{-C}
$$


Hence

$$
P^{*}(f, \mathcal{F}, \epsilon) \geq \frac{1}{k} P\left(f^{k}, \varphi_{k}, \delta\right)
$$

This gives that

$$
P^{*}(f, \mathcal{F}) \geq \frac{1}{k} P\left(f^{k}, \varphi_{k}\right)
$$

Therefore

$$
P^{*}(f, \mathcal{F}) \geq \frac{1}{2^{k}} P\left(f^{2^{k}}, \varphi_{2^{k}}\right) \quad \forall k \in \mathbb{N} .
$$

Let $t \mathcal{F}=\left\{t \varphi_{n}(x)\right\}$. Then we have

$$
P^{*}\left(f, s_{2^{k}} \mathcal{F}\right) \geq \frac{1}{2^{k}} P\left(f^{2^{k}}, s_{2^{k}} \varphi_{2^{k}}\right)=0 \quad \forall k \in \mathbb{N} .
$$

The monotone decreasing of $P^{*}(f, t \mathcal{F})$ with respect to $t$ implies that the unique root $s_{*}$ of the equation

$$
P^{*}(f, t \mathcal{F})=0
$$

satisfies

$$
s_{*} \geq s_{2^{k}} \quad \forall k \in \mathbb{N}
$$

Thus

$$
s_{*} \geq \bar{s}=\lim _{k \rightarrow+\infty} s_{2^{k}}
$$

Next we want to prove that

$$
\bar{s} \geq s_{*}
$$

For a fixed $m$,

$$
\frac{1}{2^{m}} P\left(f^{2^{m}}, s_{2^{m}} \varphi_{2^{m}}\right)=0
$$

Using the variational principle, for any $\mu \in \mathcal{M}(f) \subset \mathcal{M}\left(f^{2^{m}}\right)$, we have

$$
h_{\mu}(f)+\frac{1}{2^{m}} s_{2^{m}} \int_{M} \varphi_{2^{m}} d \mu=\frac{1}{2^{m}}\left(h_{\mu}\left(f^{2^{m}}\right)+s_{2^{m}} \int_{M} \varphi_{2^{m}} d \mu\right) \leq 0 .
$$

Letting $m \rightarrow \infty$, we have

$$
h_{\mu}(f)+\bar{s} \lim _{m \rightarrow \infty} \int_{M} \frac{1}{2^{m}} \varphi_{2^{m}} d \mu \leq 0 .
$$

Using the super-additive variational principle, we have

$$
P^{*}\left(f, \bar{s}\left\{\varphi_{n}\right\}\right) \leq 0 .
$$

Since $P\left(f, t\left\{\varphi_{n}\right\}\right)$ is strictly monotone decreasing with respect to $t$, we have

$$
s_{*} \leq \bar{s}
$$

Lemma 6.1. If $\phi_{n}(x)$ is a sub-additive sequence, then

$$
\lim _{k \rightarrow \infty} \frac{1}{2^{k}} P\left(f^{2^{k}}, \phi_{2^{k}}\right) \leq \lim _{m \rightarrow \infty} P\left(f, \frac{\phi_{2^{m}}}{2^{m}}\right) .
$$


Proof. For a fixed $k \in \mathbb{N}$, it is well known that if $E \subset M$ is an $(n, \epsilon)$-separated set of $f^{2^{k}}$, then $E$ is an $\left(n 2^{k}, \epsilon\right)$-separated set of $f$. By the definition,

$$
P\left(f^{2^{k}}, \phi_{2^{k}}\right)=\lim _{\epsilon \rightarrow \infty} \limsup _{n \rightarrow \infty} \frac{1}{n} \log \sup \left\{\sum_{x \in E} \exp \left(\hat{S}_{n} \phi_{2^{k}}(x)\right) \mid\right.
$$

$E$ is an $(n, \epsilon)$-separated set of $\left.f^{2^{k}}\right\}$,

where

$$
\left(\hat{S}_{n} \phi_{2^{k}}(x)\right)=\phi_{2^{k}}(x)+\phi_{2^{k}}\left(f^{2^{k}} x\right)+\cdots+\phi_{2^{k}}\left(f^{(n-1) 2^{k}} x\right) .
$$

Hence for a fixed $m<k$, let $2^{k}=2^{m} q+r$ and $C=\max _{x \in M} \max _{i=1, \ldots, 2^{m}} \phi_{i}(x)$. Then the sub-additivity of $\phi_{n}$ implies that

$$
\begin{aligned}
\phi_{2^{k}}(x) & \leq \frac{1}{2^{m}} \sum_{j=0}^{2^{m}-1} \sum_{i=0}^{q-2} \phi_{2^{m}}\left(f^{i 2^{m}+j}(x)\right) \\
& +\frac{1}{2^{m}} \sum_{j=0}^{2^{m}-1}\left[\phi_{j}(x)+\phi_{2^{m}-j+l}\left(f^{(q-1) 2^{m}+j}(x)\right)\right] \\
& \leq \sum_{i=0}^{2^{k}-1} \frac{1}{2^{m}} \phi_{2^{m}}\left(f^{i}(x)\right)+4 C .
\end{aligned}
$$

Thus for $1 \leq j \leq n-1$, we have

$$
\phi_{2^{k}}\left(f^{2^{k} j}(x)\right) \leq \sum_{i=0}^{2^{k}-1} \frac{1}{2^{m}} \phi_{2^{m}}\left(f^{i}\left(f^{2^{k} j}(x)\right)+4 C .\right.
$$

Hence

$$
\begin{aligned}
\hat{S}_{n} \phi_{2^{k}}(x) & =\phi_{2^{k}}(x)+\phi_{2^{k}}\left(f^{2^{k}} x\right)+\cdots+\phi_{2^{k}}\left(f^{(n-1) 2^{k}} x\right) \\
& \leq \sum_{i=0}^{n 2^{k}-1} \frac{1}{2^{m}} \phi_{2^{m}}\left(f^{i}(x)\right)+4 n C \\
& =S_{n 2^{k}}\left(\frac{1}{2^{m}} \phi_{2^{m}}\right)(x)+4 n C .
\end{aligned}
$$

This gives that

$$
P_{n}\left(f^{2^{k}}, \phi_{2^{k}}, \epsilon\right) \leq P_{n 2^{k}}\left(f, \frac{1}{2^{m}} \phi_{2^{m}}, \epsilon\right) \times e^{4 n C} .
$$

Thus

$$
\begin{aligned}
P\left(f^{2^{k}}, \phi_{2^{k}}\right) & \leq 2^{k} P\left(f, \frac{1}{2^{m}} \phi_{2^{m}}\right)+\lim _{n \rightarrow \infty} \frac{1}{n} \log e^{4 n C} \\
& =2^{k} P\left(f, \frac{1}{2^{m}} \phi_{2^{m}}\right)+4 C .
\end{aligned}
$$

Therefore

$$
\lim _{k \rightarrow \infty} \frac{1}{2^{k}} P\left(f^{2^{k}}, \phi_{2^{k}}\right) \leq P\left(f, \frac{1}{2^{m}} \phi_{2^{m}}\right) \quad \text { for all } m \in Z^{+} .
$$

Hence

$$
\lim _{k \rightarrow \infty} \frac{1}{2^{k}} P\left(f^{2^{k}}, \phi_{2^{k}}\right) \leq \lim _{m \rightarrow \infty} P\left(f, \frac{1}{2^{m}} \phi_{2^{m}}\right)
$$




\section{Lemma 6.2.}

$$
\lim _{n \rightarrow \infty} P\left(f, \frac{\phi_{2^{k}}}{2^{k}}\right) \leq P(f, \mathcal{F})
$$

Proof. Since $f: \Lambda \rightarrow \Lambda$ is an expanding map, $h_{\mu}(f)$ is an upper-semi-continuous function from $\mathcal{M}\left(\left.f\right|_{\Lambda}\right)$ to $R$. From the variational principle of topological pressure [17, we have that for every $k \in Z^{+}$there exists $\mu_{2^{k}} \in \mathcal{M}\left(\left.f\right|_{\Lambda}\right)$ such that

$$
P\left(\left.f\right|_{\Lambda}, \frac{1}{2^{k}} \phi_{2^{k}}\right)=h_{\mu_{2^{k}}}(f)+\int_{\Lambda} \frac{1}{2^{k}} \phi_{2^{k}} d \mu_{2^{k}}
$$

Since $\mathcal{M}\left(\left.f\right|_{\Lambda}\right)$ is compact, this implies that $\mu_{2^{k}}$ has a subsequence that converges to $\mu \in \mathcal{M}\left(\left.f\right|_{\Lambda}\right)$. Without loss of generality, suppose that $\mu_{2^{k}}$ converges to $\mu$. Using the sub-additivity and invariance of $\mu_{2^{k}}$, then we have for every $k \in \mathbb{N}$,

$$
h_{\mu_{2^{k}}}(f)+\int_{\Lambda} \frac{\phi_{2^{k}}(x)}{2^{k}} d \mu_{2^{k}} \leq h_{\mu_{2^{k}}}(f)+\int_{\Lambda} \phi_{1}(x) d \mu_{2^{k}} .
$$

Furthermore for fixed $s \in \mathbb{N}$, if $k>s$, from the sub-additivity and invariance of $\mu_{2^{k}}$, we have

$$
h_{\mu_{2^{k}}}(f)+\int_{\Lambda} \frac{\phi_{2^{k}}(x)}{2^{k}} d \mu_{2^{k}} \leq h_{\mu_{2^{k}}}(f)+\int_{\Lambda} \frac{\phi_{2^{s}}(x)}{2^{s}} d \mu_{2^{k}} .
$$

Since $h_{\mu}(f)$ is an upper-semi-continuous function, we have

$$
\begin{aligned}
\lim _{k \rightarrow \infty} P\left(f, \frac{\phi_{2^{k}}}{2^{k}}\right) & =\lim _{k \rightarrow \infty}\left(h_{\mu_{2^{k}}}(f)+\int_{\Lambda} \frac{\phi_{2^{k}}(x)}{2^{k}} d \mu_{2^{k}}\right) \\
& \leq \lim _{k \rightarrow \infty}\left(h_{\mu_{2^{k}}}(f)+\int_{\Lambda} \frac{\phi_{2^{s}}(x)}{2^{s}} d \mu_{2^{k}}\right) \\
& \leq h_{\mu}(f)+\int_{\Lambda} \frac{\phi_{2^{s}}(x)}{2^{s}} d \mu .
\end{aligned}
$$

Since the sequence $\left\{\int_{\Lambda} \phi_{n}(x) d \mu\right\}$ is a sub-additive sequence, we have

$$
\lim _{n \rightarrow \infty} \int_{\Lambda} \frac{\phi_{n}(x)}{n} d \mu=\inf _{n \geq 1}\left\{\int_{\Lambda} \frac{\phi_{n}(x)}{n} d \mu\right\} .
$$

The arbitrariness of $s \in \mathbb{N}$ implies that

$$
\lim _{k \rightarrow \infty} P\left(f, \frac{\phi_{2^{k}}}{2^{k}}\right) \leq h_{\mu}(f)+\lim _{s \rightarrow \infty} \int_{\Lambda} \frac{\phi_{2^{s}}}{2^{s}}(x) d \mu .
$$

Hence by the variational principle of the sub-additive topological pressure in 4], we have

$$
\lim _{k \rightarrow \infty} P\left(f, \frac{\phi_{2^{k}}}{2^{k}}\right) \leq h_{\mu}(f)+\lim _{s \rightarrow \infty} \int_{\Lambda} \frac{\phi_{2^{s}}}{2^{s}}(x) d \mu \leq P(f, \mathcal{F}) .
$$

This completes the proof of the lemma.

Theorem 6.3. The sequence $\left\{t_{2^{n}}\right\}$ is monotone, and

$$
\lim _{n \rightarrow \infty} t_{2^{n}}=t^{*}
$$

where $t^{*}$ is the unique root of the equation $P\left(f,-t\left\{\log m\left(D f^{n}(x)\right)\right\}\right)=0$. 
Proof. First we prove that the sequence $\left\{t_{2^{n}}\right\}$ is monotone decreasing. Let $\phi_{n}=$ $-\log m\left(D f^{n}(x)\right)$. For a fixed $k \in \mathbb{N}$,

$$
P_{k}(\phi,, \epsilon)=\sup \left\{\sum_{x \in E} \exp S_{n} \phi(x): E \text { is an }(n, \epsilon) \text {-separated subset of } X\right\} .
$$

For $\forall \epsilon>0$, by the uniform continuity of $f$, there exists $\delta>0$ such that if $E \subset M$ is an $(n, \epsilon)$-separated set of $f^{2^{k+1}}$, then $E$ is a $(2 n, \delta)$-separated set of $f^{2^{k}}$ and $\delta \rightarrow 0$ when $\epsilon \rightarrow 0$. Using the sub-additivity of $\phi_{n}$, the Birkhoff sum $S_{n} \phi_{2^{k+1}}$ of $\phi_{2^{k+1}}$ with respect to $f^{2^{k+1}}$ has the following property:

$$
\begin{aligned}
S_{n} \phi_{2^{k+1}}(x) & =\phi_{2^{k+1}}(x)+\phi_{2^{k+1}}\left(f^{2^{k+1}} x\right)+\cdots+\phi_{2^{k+1}}\left(f^{2^{k+1}(n-1)} x\right) \\
& \leq \phi_{2^{k}}(x)+\phi_{2^{k}}\left(f^{2^{k}} x\right)+\phi_{2^{k}}\left(f^{2^{k+1}} x\right)+\phi_{2^{k}}\left(f^{2^{k+1}} f^{2^{k}} x\right) \\
& +\cdots+\phi_{2^{k}}\left(f^{2^{k+1}(n-1)} x\right)+\phi_{2^{k}}\left(f^{2^{k+1}(n-1)} f^{2^{k}} x\right) \\
& =S_{2 n} \phi_{2^{k}}(x),
\end{aligned}
$$

where $S_{2 n} \phi_{2^{k}}(x)$ is the Birkhoff sum of $\phi_{2^{k}}$ with respect to $f^{2^{k}}$.

Thus

$$
P_{n}\left(f^{2^{k+1}}, \phi_{2^{k+1}}, \epsilon\right) \leq P_{2 n}\left(f^{2^{k}}, \phi_{2^{k}}, \delta\right) .
$$

Hence

$$
P\left(f^{2^{k+1}}, \phi_{2^{k+1}}\right) \leq 2 P\left(f^{2^{k}}, \phi_{2^{k}}\right) .
$$

Therefore if $t_{2^{k+1}}$ is the unique root of Bowen's equation $P\left(t \phi_{2^{k+1}}\right)=0$, then we have

$$
0=P\left(f^{2^{k+1}}, t_{2^{k+1}} \phi_{2^{k+1}}\right) \leq 2 P\left(f^{2^{k}}, t_{2^{k+1}} \phi_{2^{k}}\right) .
$$

The monotone decreasing of the function $P\left(f^{2^{k}}, t \phi_{2^{k}}\right)$ implies that $t_{2^{k}} \geq t_{2^{k+1}}$.

The arbitrariness of $k$ implies that the sequence $\left\{t_{2^{k}}\right\}$ is monotone decreasing. Hence the limit exists and we denote the limit of this sequence by $\bar{t}$. From the proof as above, we have

$$
\frac{P\left(f^{2^{k+1}}, \phi_{2^{k+1}}\right)}{2^{k+1}} \leq \frac{P\left(f^{2^{k}}, \phi_{2^{k}}\right)}{2^{k}} \leq \cdots \leq \frac{P\left(f^{2}, \phi_{2}\right)}{2} \leq P(f, \phi) .
$$

Next we prove that

$$
P(f, \mathcal{F}) \leq \frac{1}{k} P\left(f^{k}, \phi_{k}\right) \quad \forall k \in \mathbb{N} .
$$

For a fixed $k \in \mathbb{N}$, let $n=k m+r, 0 \leq r<k$, and let $C=\max _{x \in M} \max _{1 \leq i \leq k} \phi_{i}(x)$. For $\forall \epsilon>0$, by the uniform continuity of $f$, there exists $\delta>0$ such that if $E \subset M$ is an $(n, \epsilon)$-separated set of $f$, then $E$ is an $(m, \delta)$-separated set of $f^{k}$ and $\delta \rightarrow 0$ when $\epsilon \rightarrow 0$. Using the sub-additivity of $\phi_{n}$, we have

$$
\phi_{n}(x) \leq \phi_{k}(x)+\phi_{k}\left(f^{k}(x)\right)+\cdots+\phi_{k}\left(f^{(m-1) k}(x)\right)+\phi_{r}\left(f^{m k}(x)\right) .
$$

Thus

$$
P_{n}(f, \mathcal{F}, \epsilon) \leq P_{m}\left(f^{k}, \phi_{k}, \delta\right) \times e^{C} .
$$

Hence

$$
P(f, \mathcal{F}, \epsilon) \leq \frac{1}{k} P\left(f^{k}, \phi_{k}, \delta\right) .
$$

This gives that

$$
P(f, \mathcal{F}) \leq \frac{1}{k} P\left(f^{k}, \phi_{k}\right)
$$


Therefore

$$
P(f, \mathcal{F}) \leq \frac{1}{2^{k}} P\left(f^{2^{k}}, \phi_{2^{k}}\right) \quad \forall k \in \mathbb{N} .
$$

Let $t \mathcal{F}=\left\{t \phi_{n}(x)\right\}$. Then we have

$$
P\left(f, t_{2^{k}} \mathcal{F}\right) \leq \frac{1}{2^{k}} P\left(f^{2^{k}}, t_{2^{k}} \phi_{2^{k}}\right)=0 \quad \forall k \in \mathbb{N} .
$$

Therefore the unique root $t^{*}$ of the equation

$$
P(f, t \mathcal{F})=0
$$

satisfies

$$
t^{*} \leq t_{2^{k}} \quad \forall k \in \mathbb{N}
$$

Thus

$$
t^{*} \leq \bar{t}=\lim _{k \rightarrow+\infty} t_{2^{k}}
$$

Next we want to prove that

$$
\bar{t} \leq t^{*} .
$$

From Theorem 6.2 and Lemmas 6.1 and 6.2, we have that the sequence $\left\{\frac{1}{2^{k}} P\left(f^{2^{k}}, \phi_{2^{k}}\right)\right\}$ is monotone decreasing and it converges to $P(f, \mathcal{F})$. By the definition, it is easy to prove that

$$
0 \leq \frac{P\left(f^{2^{k}}, \bar{t} \phi_{2^{k}}\right)}{2^{k}}-\frac{P\left(f^{2^{k}}, t_{2^{k}} \phi_{2^{k}}\right)}{2^{k}} \leq\left|\bar{t}-t_{2^{k}}\right| C, \quad \forall k \in \mathbb{N},
$$

where $C=\max _{x \in M}\left|\phi_{1}(x)\right|$. Letting $k \rightarrow \infty$, we have

$$
P(f, \bar{t} \mathcal{F})=0 .
$$

Hence, we have

$$
\bar{t}=t^{*}
$$

Theorem 6.4. $t^{*}=s_{*}$.

Proof. From the theorems as above, we have that the functions

$$
P\left(f,-t\left\{\log m\left(D f^{n}(x)\right)\right\}\right)
$$

and

$$
P\left(f,-t\left\{\log \left\|D f^{n}(x)\right\|\right\}\right)
$$

coincide and both of them have unique zero points. Therefore

$$
t^{*}=s_{*} .
$$

The proof of the main theorem. From Corollary 5 and Theorem 6.4 stated above, we have

$$
\operatorname{dim}_{H} \Lambda=\underline{\operatorname{dim}}_{B} \Lambda=\overline{\operatorname{dim}}_{B}=s_{*}=t^{*} .
$$

This completes the proof of the main theorem.

Corollary 6. If $\Lambda$ is an average conformal repeller, then the Hausdorff dimension of $\Lambda$ is the zero $t^{*}$ of

$$
t \mapsto P\left(-t \frac{1}{d} \log (|\operatorname{det}(D f)|)\right),
$$

where $d=\operatorname{dim} M$ and $t \mapsto P\left(-t \frac{1}{d} \log (|\operatorname{det}| D f \mid)\right)$ is classical topological pressure. 
Proof. If $\Lambda$ is an average conformal repeller, then by Theorem 4.2, we have

$$
\lim _{n \rightarrow \infty} \frac{1}{n}\left(\log \left\|D f^{n}(x)\right\|-\log m\left(D f^{n}(x)\right)\right)=0
$$

uniformly on $\Lambda$.

On the other hand, $\log \left(m\left(D f^{n}(x)\right)\right) \leq \frac{1}{d} \log \left(\left|\operatorname{det}\left(D f^{n}(x)\right)\right|\right) \leq \log \left(\left|D f^{n}(x)\right|\right)$. Therefore

$$
\begin{aligned}
P\left(f,-t^{*}\left\{\log m\left(D f^{n}(x)\right)\right\}\right) & =P\left(f,-t^{*}\left\{\frac{1}{d} \log \left|\operatorname{det}\left(D f^{n}(x)\right)\right|\right\}\right) \\
& =P\left(f,-t^{*}\left\{\log \left\|D f^{n}(x)\right\|\right\}\right)=0 .
\end{aligned}
$$

The additivity of $\left\{\log \left\|D f^{n}(x)\right\|\right\}$ implies that

$$
P\left(f,-t^{*}\left\{\frac{1}{d} \log \left|\operatorname{det}\left(D f^{n}(x)\right)\right|\right\}\right)=P\left(f,-t^{*} \log \frac{1}{d} \mid \operatorname{det}(D f(x) \mid)=0 .\right.
$$

That is to say that $t^{*}$ is the root of the equation $P\left(-t \frac{1}{d} \log |\operatorname{det}(D f)|\right)=0$. This gives the proof of the corollary.

\section{ACKNOWLEDGEMENT}

The authors would like to thank Prof. Dejun Feng, Prof. Y. Pesin and Prof. Marcelo Viana for their discussions and suggestions. The authors would also like to thank the referees for their suggestions. The first author was partially supported by the National Science Council, R.O.C. (Contract No. NSC 95-2115-M-026-003) and the National Center for Theoretical Sciences. The second author was partially supported by NSFC(10571130), NCET, and 973 Project (2007CB814800).

\section{REFERENCES}

[1] Barreira, L.: Dimension estimates in nonconformal hyperbolic dynamics. Nonlinearity 16 (2003), no. 5, 1657-1672. MR.1999573 (2004i:37046)

[2] Barreira, L.: A non-additive thermodynamic formalism and applications to dimension theory of hyperbolic dynamical systems, Ergodic Theory Dynam. Syst. 16(1996) 871-927. MR.1417767 (98a:58124)

[3] Bowen, R.: Hausdorff dimension of quasicircles. Inst. Hautes Études Sci. Publ. Math. 50(1979) 11-25. MR.556580 (81g:57023)

[4] Cao, Yongluo, Feng, Dejun, and Huang, Wen: The thermodynamic formalism for subadditive potentials. Discrete and Continuous Dynamical Systems 20 (2008), no. 3, 639-657. MR2373208 (2008k:37072)

[5] Douady, A. and Oesterlé, J.: Dimension de Hausdorff des attracteurs. C. R. Acad. Sci. Paris 290(1980) 1135-1138. MR.585918 (82a:58033)

[6] Falconer, K.: The Hausdorff dimension of self-affine fractals. Math. Proc. Camb. Phil. Soc. 103(1988) 339-350. MR923687 (89h:28010)

[7] Falconer, K.: Dimensions and measures of quasi self-similar sets. Proc. Amer. Math. Soc. 106(1989) 543-554. MR969315 (90c:58103)

[8] Falconer, K.: Bounded distortion and dimension for non-conformal repellers. Math. Proc. Camb. Phil. Soc. 115(1994) 315-334. MR.1277063 (95g:58138)

[9] Gatzouras, D. and Peres, Y.: Invariant measures of full dimension for some expanding maps. Ergodic Theory Dynam. Systems 17 (1997), 147-167. MR1440772 (98c:58093)

[10] Hu, Huyi: Dimensions of invariant sets of expanding maps. Commum. Math. Phys. 176(1996), 307-320. MR:1374415 (96k:58135)

[11] Ledrappier, F. Some relations between dimension and Lyapounov exponents. Commum. Math. Phys. 81(1981), 229-238. MR632758(83b:58044)

[12] Pesin, Y.: Dimension Theory in Dynamical Systems. Contemporary Views and Applications (Chicago, IL: University of Chicago Press), 1997. MR1489237(99b:58003) 
[13] Ruelle, D.: Repellers for real analytic maps. Ergodic Theory Dynam. Syst. 2(1982) 99-107. MR684247 (84f:58095)

[14] Ruelle, D.: An inequality for the entropy of differential maps. Bol. Soc. Bras. De Mat. 9(1978) 83-87. MR516310 (80f:58026)

[15] Simon, K.: Hausdorff dimension of hyperbolic attractors in $R^{3}$. Fractal geometry and stochastics III, 79-92, Progr. Probab., 57, Birkhäuser, Basel (2004). MR2087133 (2005j:37029)

[16] Simon, K. and Solomyak, B.: Hausdorff dimension for horseshoes in $\mathbb{R}^{3}$. Ergodic Theory Dynam. Syst. 19(1999) 1343-63. MR1721625 (2001k:37041)

[17] Walters, P.: An introduction to ergodic theory. Springer, Berlin, 1982. MR648108(84e:28017)

[18] Zhang, Yingjie: Dynamical upper bounds for Hausdorff dimension of invariant sets. Ergodic Theory Dynam. Systems 17 (1997), no. 3, 739-756. MR1452191(98e:58111)

Department of Applied Mathematics, National Dong Hwa University, Hualien 97401, Taiwan - and - Taida Institute for Mathematical Science, National Taiwan University, TAIPEI 10617, TAIWAN

E-mail address: jcban@mail.ndhu.edu.tw

Department of Mathematics, Suzhou University, Suzhou, 215006, Jiangsu, People's Republic of China - and - Institute of Mathematics, Fudan University, Shanghai, 200433, People's Republic of China

E-mail address: ylcao@suda.edu.cn, sudacaoyongluo@gmail.com

Department of Mathematics, Michigan State University, East Lansing, Michigan 48824

E-mail address: hu@math.msu.edu 